\title{
Potential role of ACE 2 in coronavirus disease 2019 (COVID-19) prevention and management
}

\author{
Mengyuan Liu ${ }^{1,2,3}$, Ting Wang ${ }^{1,2,3}$, Yun Zhou ${ }^{1,2,3}$, Yutong Zhao ${ }^{1,2,3}$, Yan Zhang ${ }^{1,2,3}$, \\ Jianping $\mathbf{L i}^{1,2,3}$ \\ 'Department of Cardiology, Peking University First Hospital, Beijing, China; \\ ${ }^{2} \mathrm{NHC}$ Key Laboratory of Cardiovascular Molecular Biology and Regulatory Peptides, Beijing, China; \\ ${ }^{3}$ Key Laboratory of Molecular Cardiovascular Sciences of Ministry of Education, Health Science Center, Peking \\ University, Beijing, China
}

\section{ABSTRACT}

COVID-19 is the current public health threat all over the world. Unfortunately, there is no specific prevention and treatment strategy for this disease. We aim to explore the potential role of angiotensin-converting enzyme 2 (ACE2) in this regard through this literature review. As a crucial enzyme of renin-angiotensin-aldosterone system (RAAS), ACE2 not only mediates the virus entry but also affects the pathophysiological process of virus-induced acute lung injury (ALI), as well as other organs' damage. As interaction of COVID-19 virus spike and ACE2 is essential for virus infection, COVID-19-specific vaccine based on spike protein, small molecule compound interrupting their interaction, human monoclonal antibody based on receptorbinding domain, and recombinant human ACE2 protein (rhuACE2) have aroused the interests of researchers. Meanwhile, ACE2 could catalyze angiotensin II (Ang II) to form angiotensin 1-7 (Ang 1-7), thus alleviates the harmful effect of Ang II and amplifies the protection effect of Ang1-7. ACE inhibitor and angiotensin II receptor blocker (ARB) have been shown to increase the level of expression of ACE2 and could be potential strategies in protecting lungs, heart, and kidneys. ACE2 plays a very important role in the pathogenesis and pathophysiology of COVID-19 infection. Strategies targeting ACE2 and its ligand, COVID-19 virus spike protein, may provide novel method in the prevention and management of novel coronavirus pneumonia.

Key words: angiotensin-converting enzyme 2 (ACE2), coronavirus disease 2019 (COVID-19), spike protein, angiotensin II (Ang II), acute lung injury (ALI)

\section{INTRODUCTION}

From December 8, 2019, several cases of pneumonia of unknown cause have appeared in succession in Wuhan, Hubei province, China. The disease has rapidly spread from Wuhan to other areas, raising intense attention worldwide. ${ }^{[1,2]}$ By January 7, 2020, a novel coronavirus (CoV) was isolated from the throat swab sample of a patient in Wuhan by Chinese Center for Disease Control and Prevention (CDC). ${ }^{[3]}$ Subsequently, it was named as 2019$\mathrm{nCoV}$, and this coronavirus disease was named as COVID-19 by the World Health Organization. As of February 28, 2020, a total of 78,961 cases with confirmed COVID-19 had been detected in China, of whom 2,791 have died. ${ }^{[4]}$ About 4691 confirmed cases had been reported outside of China, spreading across 51 countries globally, ${ }^{[4]}$ ranking the COVID-19 the biggest public health threat all over the world.

Full-genome sequencing and phylogenic analysis revealed that COVID-19 virus belonged to the subgenus Sarbecovirus of the genus Betacoronavirus. It is a single-strand, positive-sense, RNA virus and shares $\sim 79 \%$ similarity of the genome sequence with human severe acute respiratory syndrome coronavirus(SARS$\mathrm{CoV}) \cdot{ }^{[5]}$ It was also suggested that bats might be the original host of this virus and then passed to humans through some unknown intermediate host. Human-tohuman transmission has been validated, leading to further outbreaks in other areas 
outside Wuhan. ${ }^{[6]}$ Most of patients represented with fever, cough, vomiting, diarrhea, and other symptoms, and some cases might develop severe pneumonia, acute respiratory distress syndrome (ARDS), or multiple organ failure and even die. ${ }^{[3,7]}$

As an important metalloproteinase, angiotensin-converting enzyme 2 (ACE2) was proved to mediate the infection of COVID-19 virus. ${ }^{[5]}$ Furthermore, ACE2 plays a crucial role in acute lung injury (ALI), as well as other organ damages. Therefore, interventions targeting ACE2 have aroused great interests, regarding alleviate viral infection and reduce organ injuries.

In this review, we comprehensively elucidated function of ACE2, regulation of its expression, and its crucial role in multiple organ injury such as lungs, heart, and kidneys. The novel therapeutic strategies targeting ACE2 in the recent days are also summarized, which helps better understand the potential role of ACE2 in COVID-19 and provides new insights into possible medications.

\section{A CLINICAL OVERVIEW OF CORONAVIRUS DISEASE 2019}

Early of the outbreak, most of infected patients had some connection with Huanan seafood market in Wuhan, China ${ }^{[1]}$ and most subsequent patients had an epidemiological history of traveling in Wuhan or contacting with patients with COVID-19. Asymptomatic infected individuals might also serve as the source of infection, which made the outbreak difficult to control. Inhalation and contact transmission are the two major modes of transmission. The incubation period is within 1-14 days, and most patients represented with an incubation period of 3-7 days. Common symptoms included fever $(98.6 \%)$, fatigue $(69.6 \%)$, and dry cough $(59.4 \%) .{ }^{[3,8]}$ Wang et al. summarized 138 cases of COVID-19 and demonstrated some relative common symptoms such as anorexia $(39.9 \%)$, myalgia (34.8\%), dyspnea (31.2\%), expectoration $(26.8 \%)$, pharyngalgia (17.4\%), diarrhea (10.1\%), and nausea (10.1\%). Unusual symptoms included dizziness $(9.4 \%)$, headache $(6.5 \%)$, vomiting $(3.6 \%)$, and abdominal pain $(2.2 \%)$. Severe patients usually developed dyspnea and hypoxemia 1 week later. Fatal complications included acute respiratory distress syndrome (ARDS), intractable metabolic acidosis, septic shock, and coagulation dysfunction, and some patients developed arrhythmia, acute cardiac injury, and acute kidney injury. Lymphopenia commonly occurred in patients with COVID-19, with elevated lactate dehydrogenase, hepatic enzyme, creatine kinase, and C-reactive protein. ${ }^{[8]}$ Chest computed tomographic scans usually revealed multifocal small patchy shadows and interstitial changes in the peripheral of lung and then bilateral multiple ground glass opacity. Consolidation could also be observed in severe cases. RT-PCR assays for COVID-19 using nasopharyngeal swab, sputum, serum, or stool samples of patients were available but needed repeat testing. Whole genome sequencing of the virus is available in some assigned laboratories.

As for treatment, the majority of patients received antiviral therapy (oseltamivir) and many received antimicrobial therapy (moxifloxacin, ceftriaxone, and azithromycin), and $44.9 \%$ of them received glucocorticoid therapy. High-flow oxygen therapy, noninvasive and invasive ventilation, or even extracorporeal membrane oxygenation were used for respiratory support. Some patients received renal replacement therapy. ${ }^{\left[{ }^{[8]}\right.}$ So far, none of the treatments have been proved to be efficient enough ${ }^{[9]}$ and novel strategies are needed to improve the efficacy of the therapy for COVID-19.

\section{THE POTENTIAL ROLE OF ACE2 IN THE MANAGEMENT OF CORONAVIRUS DISEASE 2019}

ACE2 was discovered in 2000 as a homologous of angiotensin-converting enzyme (ACE).$^{[10,11]}$ ACE2 is a type I transmembrane protein composed of 805 amino acids and has two domains, namely, amino- and carboxy-terminal catalytic domains. The catalytic domain contains one active site, the zinc metalloprotein domain, which is consistent with $41.8 \%$ of sequence of ACE. ${ }^{[10-12]}$ The gene encoding ACE2 is located on the X chromosome, Xp22.2..$^{[13]}$ Evidences show that ACE2 is expressed in heart, kidney, lung, liver, testis, intestine, and other tissues. ${ }^{[1]}$

\section{The Function of ACE2}

Biological functions of ACE2 could be divided into two categories: peptidase dependent and peptidase independent. The peptidase-dependent function refers to the metabolism of angiotensin I (Ang I) into angiotensin 1-9 (Ang 1-9) peptide and angiotensin II (Ang II) into angiotensin 1-7 (Ang 1-7) peptide (Figure 1). The peptidase-independent function of ACE2 refers mainly to the mediation of coronavirus infection. The peptidase-independent function also involves the absorption of amino acids in the gut. ACE2 binds with $\mathrm{B}^{0} \mathrm{AT} 1$ amino acid transporter to mediate the absorption of neutral amino acids in the intestines. ${ }^{[14]}$

\section{ACE2 as a receptor for coronavirus ACE2 as a receptor for severe acute respiratory syndrome coronavirus}

By 2003, ACE2 was identified as a functional receptor for severe acute respiratory syndrome coronavirus (SARS-CoV), which mediated the process of infection and transmission independent of its catalytic activity as 


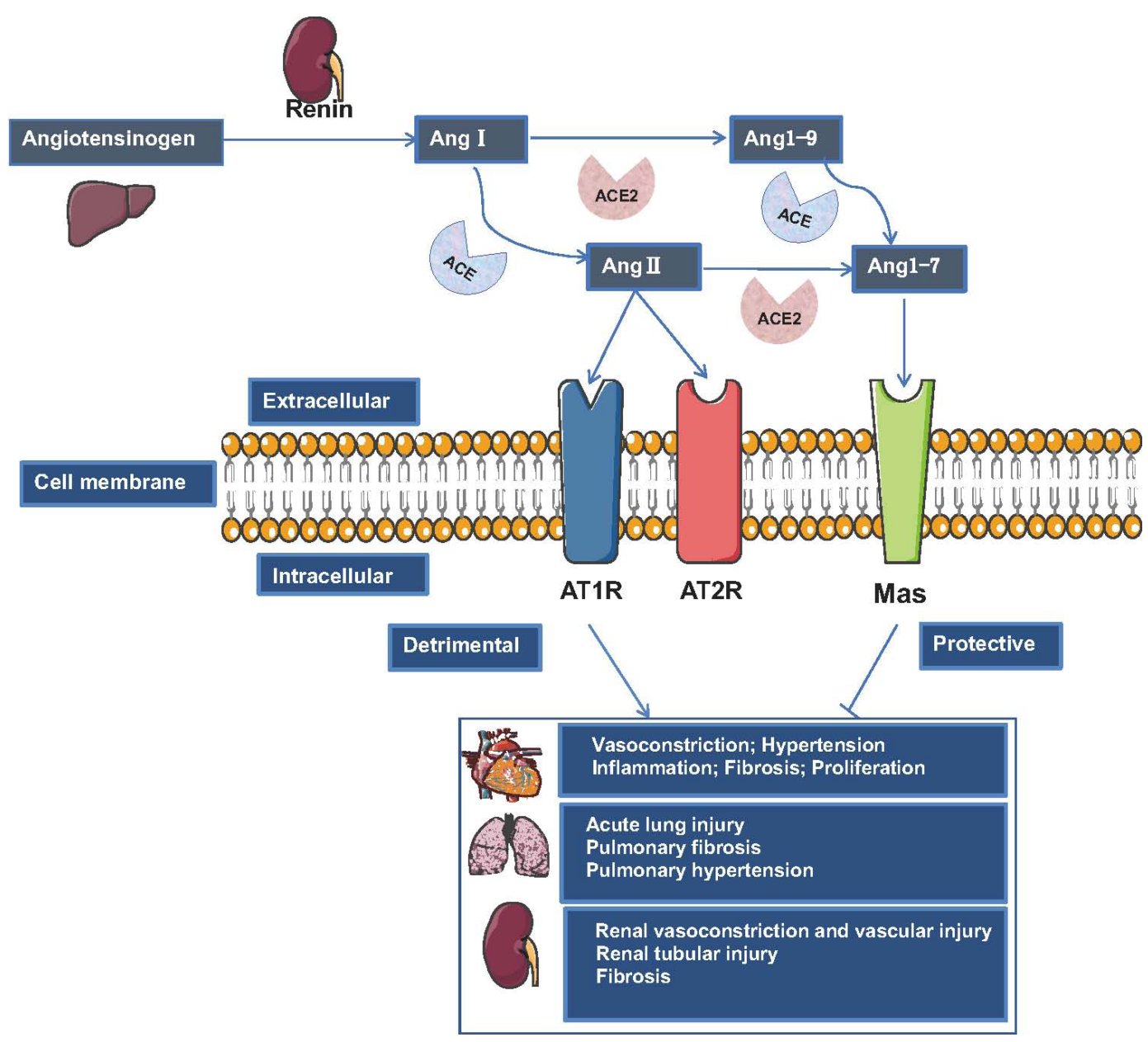

Figure 1: Schematic diagram of the role of ACE2 in renin-angiotensin-aldosterone system and its protective effect on organs. Ang I is converted to Ang II by ACE. ACE2 converts Ang II to Ang 1-7. Ang 1-7 interacts with Mas receptor to exert opposite effect to ACE/Ang I/AT1R pathway in multiple organs such as heart, lung, and kidney. ACE2 also cleaves Ang I to produce the presumably inactive Ang 1-9, and Ang1-9 can further be converted to Ang1-7 by ACE. Ang I: angiotensin I; Ang II: angiotensin II; ACE: angiotensin-converting enzyme; ACE2: angiotensin-converting enzyme 2; Ang1-7: angiotensin 1-7; Ang1-9: angiotensin 1-9; AT1R: angiotensin II type 1 receptor. AT2R: angiotensin II type 2 receptor; Mas: Mas receptor.

peptidase. ${ }^{[15]}$ The efficacy of the infection was 10 -fold increased when the SARS-CoV was applied onto the apical surface of cells that expressed ACE2. ${ }^{[16]}$ According to structural analyses, spike protein of SARS-CoV (SARS-S) contacted the apex of subunit I of the ACE2 catalytic domain but did not influence the subunit II nor occlude the active site of peptidase. Once attached to ACE2 by SARS$\mathrm{CoV}$, the ectodomain of ACE2 is cleaved, accompanied by endocytosis of transmembrane domain into the cell. Sometimes ACE2 can be internalized as an intact molecule. The internalization and virus particle-host cell fusion are essential for virus entry (Figure 2). ${ }^{[17]}$

\section{ACE2 as a receptor for COVID-19 virus}

On the basis of next-generation sequencing, the full genome sequence of the COVID-19 virushas been successfully identified. COVID-19 virus had a close relationship with two other SARS-like coronaviruses that are derived from bat, bat-SL-CoVZC45 and bat-SL-
CoVZXC21, with 88\% identity. However, COVID-19 virus has less genetical similarity with SARS-CoV (about 79\%) and MERS-CoV (about 50\%). ${ }^{[5]}$ Phylogenetic analysis indicated that COVID-19 virus was a new betacoronavirus from the subgenus Sarbecovirus. ${ }^{[5]}$ Spike protein sequences of COVID-19 virus and SARS-CoV were compared; the overall sequence similarities between the spike of these two viruses were about $76 \%-78 \%$ for the whole protein, around $73 \%-76 \%$ for the receptor-binding domain (RBD), and $50 \%-53 \%$ for the receptor-binding motif (RBM). These high similarities strongly suggested that COVID-19 virus used ACE2 as its receptor. ${ }^{[18]}$ Recently, Wrapp et al. examined a $3.5-\mathrm{A}^{\circ}$-resolution cryo-EM structure of the COVID-19 spike (S) trimer in the prefusion conformation. They discovered that COVID-19 S shared the same mechanism of triggering similar to other viruses in the Coronaviridae. To be specific, receptor binding to exposed RBDs resulted to an unstable $3 \mathrm{RBD}$ up conformation leading to shedding of S1 and refolding of S2. Biophysical 


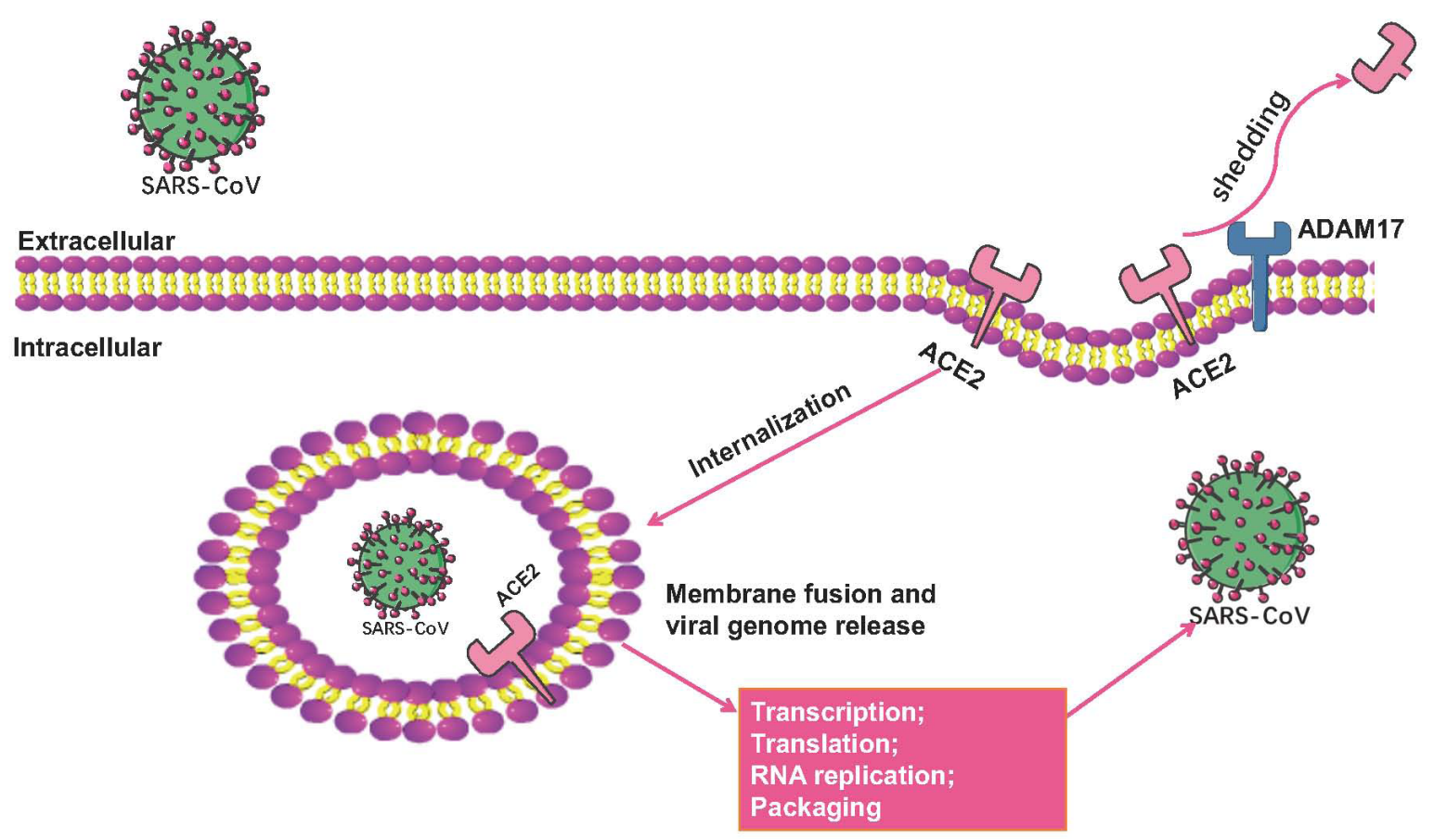

Figure 2: Schematic diagram of SARS-CoV infecting cells through ACE2. SARS-CoV binds to ACE2 for entry into cells. After the spike protein of the virus contacting with ACE2, the whole molecule or transmembrane region of ACE2 along with the virus enters the cell through endocytosis (internalization). Then membrane fusion occurs and viral RNAs are released. ADAM17 cleaves the extracellular juxta-membrane region of ACE2 (shedding). ACE2: angiotensin-converting enzyme 2; ADAM17: a disintegrin and metalloprotease 17; SARS-CoV: severe acute respiratory syndrome coronavirus.

and structural evidence showed that the COVID-19 S bound ACE2 with 10- to 20- fold higher affinity than SARS-CoV S. The high affinity of COVID-19 S for human angiotensin-converting enzyme 2 (hACE2) might facilitate it to spread from human to human. ${ }^{[19]} \mathrm{BaO}$ et al. ${ }^{[20]}$ conformed that COVID-19 virus could infect the hACE2 transgenic mice and induce typical interstitial pneumonia, whereas wild-type mice could not be infected by the virus. Moreover, the COVID-19 S protein and hACE2 receptor were discovered to colocalize in alveolar epithelial cells. ${ }^{[20]}$ These results further supported that the COVID-19 virus uses the hACE2 as a receptor for cell entry.

\section{ACE2 as a negative regulator of RAAS}

ACE2 belongs to the M2 family of metalloproteases, with their catalytic active site exposed to the extracellular surface, which promotes the metabolism of circulating peptides. ACE2 cleaves Ang I to produce the presumably inactive Ang 1-9 peptide, and Ang 1-9 can be further metabolized into Ang 1-7 by ACE or other peptidases. ${ }^{[10,11]}$ ACE2 converts Ang II to Ang 1-7 with much higher efficiency than metabolizing Ang I to generate Ang 1-9. ${ }^{[21]}$ Ang 1-7 peptide interacts with Mas receptor, a G-protein-coupled receptor, to exert vasodilation and antiproliferative effect, opposite to ACE/Ang II/AT1R pathway $^{[22-25]}$ (Figure 1). Evidences have shown that Ang
1-7 have a variety of protective effects on cardiovascular system, including anti-inflammatory, antithrombotic, antifibrosis, antihypertrophic, antiarrhythmia, and natriuretic effect. It also mediates inhibition of plaque formation and improvement of vascular dysfunction associated with metabolic syndrome. ${ }^{[2]}$ ACE2/Ang 1-7/ Mas pathway has been proved to exert beneficial effects in various animal models of hypertension, heart failure, and ischemic and non-ischemic cardiomyopathy. ${ }^{[27]}$ Besides, ACE2 can cleave an amino acid from the C-terminus of the peptides Apelin-13 in vitro, which makes Apelin-13 lose its hypotensive action. ${ }^{[21]}$

\section{The regulation of expression of ACE2 Pathophysiological and pharmacological regulation of ACE2 transcription}

Various pathological status may influence the expression of ACE2. For instance, the expression of ACE2 was downregulated in the kidneys of mice with hypertension ${ }^{13}$ and diabetes. ${ }^{[28]}$ Zisman et al. found that the expression of ACE2 was increased in failing heart ventricles of patients with idiopathic dilated cardiomyopathy. ${ }^{[29]} \mathrm{Kuba}$ et al. discovered decreased expression of ACE2 in ALI mice model. ${ }^{[30]}$ Koitka et al. illustrated that the expression of ACE2 was significantly decreased in rats with renal disease and subtotal nephrectomy. 
Transcription of ACE2 is proved to be regulated by a variety of drugs. Evidences indicated that inhibitor of renin-angiotensin-aldosterone system (RASI) such as angiotensin-converting enzyme inhibitor (ACEI) or angiotensin II type 1 receptor (AT1R) antagonist could upregulate the mRNA level of ACE2. Ishiyama et al. ${ }^{[31]}$ examined the effect of myocardial ischemia on the expression of cardiac ACE2 mRNA using rat model of myocardial infarction (MI). The results showed that although cardiac ACE2 mRNA did not change in MI vehicle-treated rat, however, both losartan and olmesartan elevated ACE2 mRNA by an average of $97 \%$ and $42 \%$, respectively, after treatment for 28 days, indicating that cardioprotective effect of AT1R antagonist partially attributed to ACE2. Ferrario et al. showed that after the treatment with lisinopril or losartan for 12 days, ACE2 mRNA level of heart increased by 4.7fold or 2.8-fold in rats, respectively. Rats medicated with losartan alone or in combination with lisinopril showed significant increase in the activity of ACE2. ${ }^{[32]}$ Keidar et al. demonstrated that mineralocorticoid receptor blockade (spironolactone) significantly increased the activity of ACE2 and the expression of mRNA in macrophages obtained from 10 patients with chronic heart disease by $300 \%$ and $654 \%$, respectively. This effect of spironolactone might be mediated by the inhibition of Ang II/NF- $x$ B pathway and reduction of oxidative stress. ${ }^{[33]}$ Treatment of Vero E6 cells with IL-4 and IFN- $\gamma$ downregulated ACE2 mRNA levels and the expression of ACE2 on cell surface and inhibited the susceptibility to SARS-CoV replication partly through downregulating ACE2. ${ }^{[34]}$

\section{ACE2 shedding and internalization}

Since the wide spread of SARS-CoV in 2003, ACE2 was identified as a SARS-CoV receptor. After the spike protein of the virus contacts ACE2, the whole molecule or transmembrane region of ACE2 along with the virus enters the cell through endocytosis (internalization) with the help of clathrin. Spike is activated by trypsin or furin, which mediates membrane fusion, and viral RNAs are released into intracellular space; then SARS infection is established. ${ }^{[15]}$ ADAM17, a transmembrane proteinase, can cleave the extracellular juxta-membrane region of ACE2; then the catalytically active ectodomain was released into the extracellular milieu. ${ }^{[35]}$ This process is nominated as shedding (Figure 2). Although the role of ACE2 shedding remains unclear, some studies indicated that shedding might be associated with cellular entry and replication of SARS-CoV, because an ADAM17 inhibitor restrains SARS-CoV replication in vitro. ${ }^{[36]}$ In summary, ACE2 internalization and shedding reduced the expression of ACE2 on the cell surface.

\section{THE ROLE OF ACE2 AS A NEGATIVE REGULATOR FOR RAAS IN MULTIPLE ORGAN INJURY}

\section{Acute lung injury}

The role of RAAS in the pathogenesis of acute lung injury

Acute respiratory distress syndrome (ARDS) is the most severe form of ALI and may be life-threatening. It is characterized as diffuse damage to alveolar capillary, triggered by multiple diseases such as severe pneumonia, aspiration, sepsis, and trauma. Pathological findings include increased lung permeability; edema fluid rich in neutrophils, macrophages, and protein in the alveolar spaces; and the formation of hyaline membranes. ${ }^{[37,38]}$ Since the outbreak of SARS-CoV in 2003, the role of RAAS in ARDS/ALI has attracted extensive attentions. In an animal model of ALI by aspiration of gastric contents, acid aspiration markedly increased Ang II levels in plasma and lungs of wild-type mice, but genetic inactivation of ACE remarkably decreased Ang II levels both in plasma and lungs in the same acid injury model. ${ }^{[39]} \mathrm{A}$ latest research by Liu et al. also showed that patients infected by COVID-19 represented with increased levels of Ang II. ${ }^{[40]}$ Compared with wild-type mice, $A C E-/-$ mice were protected against ALI caused by acid aspiration to some extent, and the protection effect reduced to a lesser extent in $A C E+/$ - mice; meanwhile, genetic deficiency of AT1a receptor in Agtr1a-/- mice also largely improved the lung function compared with wild-type mice, indicating a mediation role of ACE/ Ang II/AT1R in the ALI model. ${ }^{[39]}$ Ang II levels in the lung tissue were markedly elevated in mice treated with Spike-Fc of SARS-CoV. Furthermore, the inhibition of AT1R alleviated pulmonary edema and ALI in Spike-Fctreated mice, indicating that deregulation of RAAS was a crucial mechanism in ALI induced by SARS-CoV spike. ${ }^{[30]}$ Compared with a control cohort, patients with ARDS showed an increased frequency of $\mathrm{D} / \mathrm{D}$ genotype in $A C E$ gene. Besides, the $A C E \mathrm{D} / \mathrm{D}$ allele was associated with higher mortality in the ARDS group compared with I/I allele. These results demonstrated that the ACE insertion/ deletion (I/D) polymorphism was related to susceptibility and prognosis of ARDS. ${ }^{[41]}$ In summary, ACE/Ang II / AT1R signaling might mediate the pathogenesis of ALI.

Imbalance of ACE/Ang II/AT1R and ACE2/Ang 1-7/ Mas receptor signaling aggravates acute lung injury SARS-CoV infection of wild-type mice resulted in markedly decreased expression of ACE2 in the lung, but the expression of ACE in the lung was not changed obviously. By using recombinant SARS-CoV surface-spike protein, a crucial ligand for ACE2 binding, the expression of ACE2 on the cell surface was also downregulated in cell 
lines. ${ }^{[30]}$ Decreased expression of ACE2 would exacerbate ALI induced by a variety of causes, including coronavirus infection. ${ }^{[30]}$ In animal model of ALI, acid-treated ACE2 knockout mice presented with notably greater lung elastance, worsened oxygenation, massive lung edema, increased inflammatory infiltration, and formation of hyaline membrane, compared with acid-treated wild-type mice. The deleterious effect of ACE2 gene deficiency on sepsis-induced ALI was also proved, and ACE2 gene deficiency also increased mortality. ${ }^{[39]}$ In order to examine if loss of ACE2 is essential for pathogenesis of ALI, Imai et al. performed a rescue experiment by injecting rhuACE2; they found out that rhuACE2 decreased the degree of ALI in acid-treated ACE2 knockout mice. The protective effect of rhuACE2 could also be observed when injected to acid-treated wild-type mice. However, catalytically inactive ACE2 protein (mut-rhuACE2) could not reverse the severe lung phenotype of $A C E 2$ knockout mice or attenuate the severity of ALI in wild-type mice after acid instillation. ${ }^{[3]]}$ These findings indicated that the catalytic activity of ACE2 could protect lung from ALI directly.

Furthermore, a significant elevation in Ang II levels of lungs and plasma of acid-treated ACE2 knockout mice was observed compared with control wild-type mice. Treatment with rhuACE2 attenuated ALI as well as reduced Ang II levels in the lungs of acid-treated mice. Compared with ACE2 knockout mice, inactivation of $A C E$ on an $A C E 2$ knockout background attenuated the clinical and histological changes of ALI induced either by acid aspiration or endotoxin. Inhibition of AT1R pharmacologically alleviated the severity of ALI induced by acid in ACE2 knockout mice, but no effect was observed with the inhibition of AT2R. ${ }^{[39]}$ These data suggested that ACE2 might exerted its protective role in ALI through downregulating ACE/Ang II/AT1R.

Except for type-1 and type-2 pneumocytes and vascular endothelial cell that are susceptible to SARS-CoV, evidence has shown that lung stem/progenitor cells, which are able to differentiate into type- 2 and type- 1 pneumocytes, may be infected by SARS-CoV through ACE2. This process may impair lung repairment and cause continuous damage to lung tissues. ${ }^{[42]}$

\section{Heart failure and cardiac injury}

ACE2/Ang1-7/Mas plays a considerable role in the maintenance of cardiovascular homeostasis. Ang II induces myocardial hypertrophy, fibrosis, and diastolic dysfunction, whereas ACE2 exerts vasodilatory and antiproliferative effect by degrading Ang II to generate Ang1-7. ${ }^{[3-45]}$ Crackower et al. first suggested that ACE2 played an important role in the regulation of cardiac function in vivo. ACE2 null mice showed impaired cardiac contractility, accompanied by the decrease in blood pressure. Abnormality of cardiac structure could also be observed, with ventricular dilatation and thinning of the ventricular wall. ${ }^{\left[{ }^{13]}\right]}$ Yamamoto et al. performed transverse aortic constriction (TAC) in $A C E 2(-/ y)$ mice to investigate the role of ACE2 in response to pressure overload. Shamoperated $A C E 2(-/ y)$ mice exhibited normal cardiac function and morphology, whereas $A C E 2(-/ y)$ mice developed cardiac hypertrophy and dilatation. ${ }^{\left[{ }^{[6]}\right]}$ Treatment with AT1 receptor blockers or double knock-out mice of $A C E$ and $A C E 2$ genes reversed the cardiac phenotype in ACE2 knock-out mice. ${ }^{[13,46,47]}$ These results indicated that the balance between ACE and ACE2 regulated Ang II levels, contributing to affecting heart function through AT1 receptor.

The loss of ACE2 enhances susceptibility of heart to some pathological conditions. For instance, in MI mice model induced by left anterior descending artery ligation, the expression of ACE2 was increased in the affected myocardium in wild-type mice. ACE2 null mice were more susceptible to MI and suffered from increased infarct size, more obvious ventricular remodeling, and increased mortality. The mechanism of this phenomenon might include increased cardiac Ang II levels, decreased Ang 1-7 levels, and increased reactive oxygen species, matrix metalloproteinase (MMP)2 levels, MMP9 levels, and neutrophilic infiltration. Treatment with AT1 receptor blocker in $A C E 2(-/ y)-M I$ mice alleviated this process. ${ }^{[4]}$ So, ACE2 may minimize the adverse myocardial injury and adverse ventricular remodeling by antagonizing ACE/Ang II/AT1R.

SARS-CoV induced ACE2-dependent myocardial infection and decreased the expression of ACE2 in cardiomyocytes in mice. Patients with SARS represented with cardiac symptoms including arrhythmia, sudden cardiac death, and systolic and diastolic dysfunction. RNA of SARS-CoV was detected from $35 \%$ of autopsy heart samples from patients who died during the Toronto SARS outbreak. In patients with myocardial infection of SARS-CoV, macrophage infiltration increased significantly, and there were signs of myocardial injury. The presence of SARS-CoV in myocardium was also related to a significant decrease in the expression of ACE2. ${ }^{[49]}$ These results suggested that the downregulation of the expression of ACE2 caused by SARS-CoV infection might be an important mechanism of myocardial injury.

\section{Kidney disease}

ACE2 is localized mainly in the apical membrane of the epithelial cells in the proximal tubules, colocalizing with ACE. ${ }^{[50,51]}$ ACE2 also exists in glomerular podocytes, mesangial cells, and renal vascular system. ${ }^{[50]}$ In the kidney, 
ACE/Ang II/AT1R axis and ACE2/Ang (1-7)/Mas receptor axis are antagonistic to each other. The balance between them is of great significance for the maintenance of normal renal physiology. ACE/Ang II/AT1R axis promotes sodium reabsorption and vasoconstriction and induces cell proliferation, inflammation, oxidative stress, and fibrosis. ACE2 degrades Ang II, generating Ang 1-7, which interacts with Mas receptor, counteracting the harmful effects mediated by Ang II. ${ }^{[52]}$ ACE/ACE2 ratio is upregulated in many pathological conditions, including diabetes, ${ }^{[51]}$ hypertension, ${ }^{[53,54]}$, hypertensive nephrosclerosis, ${ }^{[5]}$ and diabetic nephropathy. ${ }^{[56]} \mathrm{Ye}$ et al. studied the expression of ACE and ACE2 in the kidney of mice model of obesity, diabetes, and dyslipidemia (db/ db). ${ }^{[50]}$ They found that in the early stage of diabetes, the expression of ACE2 in the renal cortex of $\mathrm{db} / \mathrm{db}$ mice increased, whereas the expression of ACE decreased. This pattern of increased expression of ACE2 may be a renal protective response in the early stage of diabetes, but with the progress of the disease, the expression level and activity of ACE2 may be reduced. At the same time, some studies showed that in $\mathrm{db} / \mathrm{db}$ mice, kidney ACE2 shedding to the urine increased, ${ }^{[57]}$ which might be due to the upregulation of ADAM17 mediated by hyperglycemia. ${ }^{[58]}$ Treating with ACE2 inhibitor, MLN-4760, led to the increase of proteinuria and aggravation of pathological changes in the kidneys of $\mathrm{db} / \mathrm{db}$ diabetic mice and streptozotocin-induced diabetic mice. These pathological changes can be reversed by using AT1 receptor blocker. ${ }^{[0,59]}$. The possible mechanism of Ang II/ AT1R pathway induced renal damage may be that the combination of Ang II and AT1R activates nicotinamide adenine dinucleotide phosphatase (NADPH), increases the oxidative stress response in the kidney, and leads to renal vasoconstriction, vascular damage, renal tubular damage, and fibrosis. ${ }^{[60]}$ Therefore, we concluded that the decreased expression of ACE2 promoted the occurrence and development of renal diseases and ACE2 played a protective role in kidney by reducing the level of Ang II.

\section{POTENTIAL STRATEGIES IN PREVENTION AND MANAGEMENT OF CORONAVIRUS DISEASE 2019 BASED ON ACE2}

To date, there is no specific medication against COVID-19. The main therapeutic strategies are limited to symptomatic treatment and organ support for severe patients. ${ }^{[61]}$ Multiple therapies targeting COVID-19 and regulating immune function of the host have attracted wide attention. ${ }^{[61]}$ Thanks to the dual role of ACE2 in virus infection and RAAS system, medications targeting ACE2 and its ligandCOVID-19 virus spike protein are under investigation, which may inhibit virus infection and protect the host from organ damage.

\section{Inhibition for virus infection Development of COVID-19 specific vaccine}

The roles of $\mathrm{S}$ protein in virus entry indicated that vaccines based on $S$ protein might induce antibodies to prevent virus from binding and fusing with target cell or neutralize virus infection. ${ }^{[32]}$ These hypotheses were validated in SARS-CoV a decade ago. ${ }^{[2,62]}$ Yang et al. showed that a DNA vaccine encoding full-length $\mathrm{S}$ protein could effectively induce T-cell and neutralizing-antibody response in mice model. ${ }^{\left[{ }^{[3]}\right]}$ Attenuated modified virus encoding full-length $S$ protein of SARS-CoV ${ }^{[6,65]}$ or its trimer ${ }^{[6,667}$ were used as vaccination, and it could elicit S-specific neutralizing antibodies and protective immunity in various animal models. These results indicated that full-length $\mathrm{S}$ protein was highly immunogenic and could induce protection against virus infection, justifying the rationale that full-length $\mathrm{S}$ protein could be an ideal target for vaccine development.

Vaccines based on the RBD of SARS-CoV S protein have also been developed. Recombinant SARS-CoV RBD antigen was strongly reactive with the neutralizing antibodies in the antisera of animal models immunized with inactivated SARS- CoV. ${ }^{[68]}$ The RBD highly reacted with the antisera of patients with SARS. ${ }^{\left[{ }^{[9]}\right.}$ Immunization of mice with RBD-Fc or an adeno-associated virus (AAV)based vaccine containing RBD could induce protective immunity against SARS-CoV ${ }^{[70-74]}$. Immunization with an RBD-based vaccine elicited both humoral and cellular immune responses against SARS-CoV ${ }^{[75,76]}$, so RBD is a crucial target for vaccine design.

COVID-19 virus used a glycosylated, homotrimeric class I fusion spike (S) protein to enter into host cells. Once the S1 subunit binds to the receptor of host cell, the prefusion trimer would be destabilized, leading to shedding of the S1 subunit and transition of the S2 subunit to a highly stable post-fusion conformation. ${ }^{[19,77-79]}$ The structural rearrangement of $\mathrm{S}$ protein promoted to membrane fusion. On the basis of essential function of $\mathrm{S}$ protein for the entry of COVID-19 virus and highly immunogenic property, it was a potential target for vaccine development. Discovering the atomic-level structure of COVID-19 S protein will support precision vaccine design. ${ }^{[19]}$

\section{Interruption of interaction between spike protein and ACE2}

A peptide overlapping the RBD sequence blocked the RBD-ACE2 interaction, restraining SARS-CoV entry in vitro. ${ }^{[80]}$ The evidence from animal models of MERS showed the capacity of RBD peptide to inhibit virus entry and stimulate immune response. ${ }^{[80]}$ Similarly, peptides 
derived from RBD that blocked RBD-ACE2 binding may be developed as novel medication against COVID-19 infection. Furthermore, the attachment of an Fc fragment to RBD protein would extend its lifespan in circulation, ${ }^{[81]}$ but it should not affect the enzymatic activity of ACE2.

A polypeptide containing two RBD-binding motifs of ACE2 had a potent inhibitory effect on SARS pseudovirus infection in HeLa cells that expressed ACE2. ${ }^{[82]}$ Soluble human ACE2 ectodomain could combine with the spike protein and thus specifically block SARS-S-dependent virus entry in vitro. ${ }^{[83]}$ The affinity of soluble ACE2 for the spike protein of SARS was found to be $1.70 \mathrm{nM}$. An Fc domain could also be attached to soluble ACE2 for prolonged lifespan, which was proved to be effective in binding SARS in vitro ${ }^{[81]}$ Likewise, the soluble ACE2 may be a potential drug to block COVID-19 virus entry into host cells.

Small molecule compounds such as SSAA09E2 \{N-[[4(4-methyl- piperazin-1-yl)phenyl]methyl]-1,2-oxazole-5carboxamide $\}$ and NAAE (N-(2-aminoethyl)-l-aziridineethanamine) were discovered as SARS viral entry inhibitors. ${ }^{[8,85]}$ SSAA09E2 and NAAE exerted their effect by blocking early interactions of SARS-S with ACE2. Furthermore, NAAE not only blocked S-protein-induced virus-host cell fusion but also inhibited the catalytic activity of ACE2, leading to a potential risk for hypertension. Though SARS-CoV and COVID-19 virus share ACE2 as their receptor, the effect of these compounds on COVID-19 infection remains unknown.

Chloroquine was discovered to inhibit SARS-CoV infection by interfering with ACE2 in cell culture. ${ }^{[86]}$ Recently, chloroquine was discovered to inhibit COVID-19 infection at low-micromolar concentration in vitro, which could function at both entry and post-entry stages of infection. ${ }^{[87]}$ A number of clinical trials have been conducted in China to test the efficacy and safety of chloroquine in the treatment of COVID-19. The results from more than 100 patients demonstrated that chloroquine phosphate was superior to the control group in inhibiting the exacerbation of pneumonia and shortening the disease course. ${ }^{[88]}$ Evidences showed that emodin and promazine blocked the interaction between the S protein of SARS-CoV and ACE2. ${ }^{[89,90]}$ So, they may be able to inhibit COVID-19 infection, although further investigation is warranted.

\section{Human monoclonal antibody for COVID-19}

Tian et al. ${ }^{\left[{ }^{11]}\right.}$ found that SARS-CoV-specific human monoclonal antibody CR3022 was capable of binding with RBD of COVID-19 virus. Epitope of CR3022 and ACE2 binding site in COVID-19 RBD did not overlap. However, the other potent specific neutralizing antibodies, such as m396 and CR3014, targeting the ACE2 binding site of SARS-CoV could not bind to COVID-19 S protein. Thus, CR3022 is a potential therapeutic choice for COVID-19 infection, alone or combined with other neutralizing antibodies. However, there is still a long way to go, because the candidate antibody is needed to be further tested in vivo. Besides, multiple isolates in the population are required in order to examine the breadth of coverage of the antibody. ${ }^{[81]}$

\section{Reducing the organ damage by adjusting the balance between ACE and ACE2}

Injection of rhuACE2 decreased the degree of acidinduced ALI in mice model. ${ }^{[39]}$ Recombinant ACE2 protein also largely improved the respiratory failure of ARDS in piglets. ${ }^{[2]}$ A highly soluble and glycosylated rhuACE2 (GSK2586881) was injected to healthy human volunteers. Plasma Ang II levels were decreased and this effect was dose dependent, reaching the largest effect after a single dose of $100-400 \mu \mathrm{g} / \mathrm{kg}$ of rhuACE2. Ang1-7 levels increased after using 100- and $200-\mu \mathrm{g} / \mathrm{kg}$ dose of rhuACE2. Besides, rhACE2 was well tolerated in human because blood pressure and heart rate were not markedly affected. ${ }^{\left[{ }^{[3]}\right]}$ Therefore, soluble human ACE2 may be an alternative choice for COVID-19 treatment. Unfortunately, in patients with ALI and ARDS, administration of rhuACE2 (GSK2586881) (0.4 mg/kg bid) intravenously for 3 days did not improve the oxygenation index but increase the surfactant protein D levels. ${ }^{[94]}$

Inhibition of the AT1R alleviated ALI and pulmonary edema in SARS-CoV Spike-Fc-treated mice. ${ }^{[30]}$ Losartan, an inhibitor of AT1R, proved to attenuate inflammatory response and lung injury in an LPS-induced ALI rat model. ${ }^{\left[{ }^{5]}\right]}$ Administration of Ang 1-7 was found to decrease cell infiltrates, fibrosis, and ALI in acid-induced ALI rat model ${ }^{[96]}$ and ventilator-induced ALI mice model. ${ }^{[97]}$ ACE2 activators, Ang 1-7, and Mas agonists were proved to reduce pulmonary vessel wall thickness, inflammation and fibrosis and attenuate right ventricular systolic pressure and fibrosis in pulmonary arterial hypertension models. ${ }^{[98,99]}$ On the basis of the protective role of ACE2 in multiple systems, and antagonism between ACE2 and ACE, therapeutic strategies targeting the balance between ACE and ACE2 may alleviate multiple organs damage caused by COVID-19.

\section{VIEWS ON THE USAGE OF ACEI/ ARB IN INFECTED PATIENTS WITH HYPERTENSION}

As ACE2 is recognized as COVID-19 virus receptor and its expression can be upregulated by ACEI or ARB, some hypotheses have been proposed, suggesting that ACEI/ 
ARB may increase the risk of viral infection. However, there has been no evidence that the use of such drug enhances the rate of infection and mortality to date. Besides, ACE2 may alleviate pathophysiological process of ALI based on previous animal models. Therefore, the exact influence of ACEI or ARB on COVID-19 infection, course of disease, and prognosis remains unknown. So, in our opinion, we do not recommend patients to discontinue or change medications until the conclusive evidences come out.

\section{CONCLUSIONS}

ACE2, as a crucial enzyme of RAAS, not only mediates the virus entry but also exerts protective role in the pathophysiological process of virus-induced ALI. To date, what is the sequential role of ACE2 in the whole disease process remains unclear. Real-world data and highquality clinical trials are warranted to answer the question. However, based on its important role in the pathogenesis and pathophysiology of the disease, ACE2 has motivated extensive interests and strategies targeting ACE2 and its ligand-COVID-19 spike protein and may provide novel method in the prevention and management of COVID-19.

\section{Conflicts of Interest}

The authors have no conflicts of interest to disclose.

\section{REFERENCES}

1. Huang C, Wang Y, Li X, Ren L, Zhao J, Hu Y, et al. Clinical features of patients infected with 2019 novel coronavirus in Wuhan, China. Lancet 2020;395:497-506.

2. Lu H, Stratton CW, Tang YW. Outbreak of pneumonia of unknown etiology in Wuhan, China: The mystery and the miracle. J Med Virol 2020;92:401-2.

3. Chen N, Zhou M, Dong X, Qu J, Gong F, Han Y, et al. Epidemiological and clinical characteristics of 99 cases of 2019 NCP in Wuhan, China: a descriptive study. Lancet 2020;395:507-13.

4. WHO. Coronavirus disease 2019 (COVID-19) situation reports-39. Feb 28, 2020. Available from: https://www.who.int/docs/defaultsource/coronaviruse/situation-reports/20200228-sitrep-39-covid-19. pdf?sfvrsn=5bbf3e7d_4. (Accessed Feb 28, 2020)

5. Lu R, Zhao X, Li J, Niu P, Yang B, Wu H, et al. Genomic characterisation and epidemiology of 2019 novel coronavirus: implications for virus origins and receptor binding. Lancet 2020;395:565-74.

6. Chan JF, Yuan S, Kok KH, To KK, Chu H, Yang J, et al. A familial cluster of pneumonia associated with the 2019 novel coronavirus indicating person-to-person transmission: a study of a family cluster. Lancet. 2020;395:514-23.

7. Yang Y, Lu Q, Liu M, Wang Y, Zhang A, Jalali N, et al. Epidemiological and clinical features of the 2019 novel coronavirus outbreak in China. BMJ 2020:[Epub ahead of print]

8. Wang D, Hu B, Hu C, Zhu F, Liu X, Zhang J, et al. Clinical Characteristics of 138 Hospitalized Patients With 2019 Novel Coronavirus-Infected Pneumonia in Wuhan, China. JAMA 2020:[Epub ahead of print]

9. de Wit E, van Doremalen N, Falzarano D, Munster VJ. SARS and
MERS: recent insights into emerging coronaviruses. Nat Rev Microbiol 2016;14:523-34.

10. Donoghue M, Hsieh F, Baronas E, Godbout K, Gosselin M, Stagliano N, et al. A novel angiotensin-converting enzyme-related carboxypeptidase (ACE2) converts angiotensin I to angiotensin 1-9. Circ Res 2000;87:E1-9.

11. Tipnis SR, Hooper NM, Hyde R, Karran E, Christie G, Turner AJ. A human homolog of angiotensin-converting enzyme. Cloning and functional expression as a captopril-insensitive carboxypeptidase. J Biol Chem 2000;275:33238-43.

12. Turner AJ, Hooper NM. The angiotensin-converting enzyme gene family: genomics and pharmacology. Trends Pharmacol Sci 2002;23:177-83.

13. Crackower MA, Sarao R, Oudit GY, Yagil C, Kozieradzki I, Scanga SE, et al. Angiotensin-converting enzyme 2 is an essential regulator of heart function. Nature 2002;417:822-8.

14. Camargo SM, Singer D, Makrides V, Huggel K, Pos KM, Wagner CA, et al. Tissue-specific amino acid transporter partners ACE2 and collectrin differentially interact with hartnup mutations. Gastroenterology 2009;136:872-82.

15. Li W, Moore MJ, Vasilieva N, Sui J, Wong SK, Berne MA, et al. Angiotensin-converting enzyme 2 is a functional receptor for the SARS coronavirus. Nature 2003;426:450-4.

16. Tseng CT, Tseng J, Perrone L, Worthy M, Popov V, Peters CJ. Apical entry and release of severe acute respiratory syndrome-associated coronavirus in polarized Calu-3 lung epithelial cells. J Virol 2005;79:9470-9.

17. Li F, Li W, Farzan M, Harrison SC. Structure of SARS coronavirus spike receptor-binding domain complexed with receptor. Science 2005;309:1864-8.

18. Wan Y, Shang J, Graham R, Baric RS, Li F. Receptor recognition by novel coronavirus from Wuhan: An analysis based on decade-long structural studies of SARS. J Virol 2020:[Epub ahead of print]

19. Wrapp D, Wang N, Corbett KS, Goldsmith JA, Hsieh CL, Abiona O, et al. Cryo-EM structure of the 2019-nCoV spike in the prefusion conformation. Science 2020;367:1260-3.

20. Bao L, Deng W, Huang B, Gao H, Ren L, Wei Q, et al. The pathogenicity of SARS-CoV-2 in hACE2 transgenic mice. bioRxiv 2020; February 28 , 2020. [Epub ahead of print]

21. Vickers C, Hales P, Kaushik V, Dick L, Gavin J, Tang J, et al. Hydrolysis of biological peptides by human angiotensin-converting enzyme-related carboxypeptidase. J Biol Chem 2002;277:14838-43.

22. Santos RA, Simoes e Silva AC, Maric C, Silva DM, Machado RP, de Buhr I, et al. Angiotensin-(1-7) is an endogenous ligand for the G proteincoupled receptor Mas. Proc Natl Acad Sci 2003;100:8258-63.

23. Keidar S, Kaplan M, Gamliel-Lazarovich A. ACE2 of the heart: From angiotensin I to angiotensin (1-7). Cardiovasc Res 2007;73:463-9.

24. Raizada MK, Ferreira AJ. ACE2: a new target for cardiovascular disease therapeutics. J Cardiovasc Pharmacol 2007;50:112-9.

25. Alenina N, Xu P, Rentzsch B, Patkin EL, Bader M. Genetically altered animal models for Mas and angiotensin-(1-7). Exp Physiol 2008;93:528-37.

26. Te Riet L, van Esch JH, Roks AJ, van den Meiracker AH, Danser AH. Hypertension : renin-angiotensin-aldosterone system alterations. Circ Res 2015;116:960-75.

27. Patel VB, Zhong JC, Grant MB, Oudit GY. Role of the ACE2/Angiotensin 1-7 Axis of the Renin-Angiotensin System in Heart Failure. Circ Res 2016;118:1313-26.

28. Tikellis C, Johnston CI, Forbes JM, Burns WC, Burrell LM, Risvanis J, et al. Characterization of renal angiotensin-converting enzyme 2 in diabetic nephropathy. Hypertension 2003;41:392-7.

29. Zisman LS, Keller RS, Weaver B, Lin Q, Speth R, Bristow MR, et al. Increased angiotensin-(1-7)-forming activity in failing human heart ventricles: evidence for upregulation of the angiotensin-converting enzyme Homologue ACE2. Circulation 2003;108:1707-12.

30. Kuba K, Imai Y, Rao S, Gao H, Guo F, Guan B, et al. A crucial role of angiotensin converting enzyme 2 (ACE2) in SARS coronavirus-induced lung injury. Nat Med 2005;11:875-9. 
31. Ishiyama Y, Gallagher PE, Averill DB, Tallant EA, Brosnihan KB, Ferrario $\mathrm{CM}$. Upregulation of angiotensin-converting enzyme 2 after myocardial infarction by blockade of angiotensin II receptors. Hypertension 2004;43:970-6.

32. Ferrario CM, Jessup J, Chappell MC, Averill DB, Brosnihan KB, Tallant EA, et al. Effect of angiotensin-converting enzyme inhibition and angiotensin II receptor blockers on cardiac angiotensin-converting enzyme 2. Circulation 2005;111:2605-10.

33. Keidar S, Gamliel-Lazarovich A, Kaplan M, Pavlotzky E, Hamoud S, Hayek T, et al. Mineralocorticoid receptor blocker increases angiotensinconverting enzyme 2 activity in congestive heart failure patients. Circ Res 2005;97:946-53.

34. de Lang A, Osterhaus AD, Haagmans BL. Interferon-gamma and interleukin-4 downregulate expression of the SARS coronavirus receptor ACE2 in Vero E6 cells. Virology 2006;353:474-81.

35. Wang H, Yang P, Liu K, Guo F, Zhang Y, Zhang G, et al. SARS coronavirus entry into host cells through a novel clathrin- and caveolae-independent endocytic pathway. Cell Res 2008;18:290-301.

36. Haga S, Yamamoto N, Nakai-Murakami C, Osawa Y, Tokunaga K, Sata T, et al. Modulation of TNF-alpha-converting enzyme by the spike protein of SARS-CoV and ACE2 induces TNF-alpha production and facilitates viral entry. Proc Natl Acad Sci USA 2008;105:7809-14.

37. Nagase T, Uozumi N, Ishii S, Kume K, Izumi T, Ouchi Y, et al. Acute lung injury by sepsis and acid aspiration: a key role for cytosolic phospholipase A2. Nat Immunol 2000;1:42-6.

38. Ware LB, Matthay MA. The acute respiratory distress syndrome. N Engl J Med 2000;342:1334-49.

39. Imai Y, Kuba K, Rao S, Huan Y, Guo F, Guan B, et al. Angiotensinconverting enzyme 2 protects from severe acute lung failure. Nature 2005;436:112-6.

40. Liu Y, Yang Y, Zhang C, Huang F, Wang F, Yuan J, et al. Clinical and biochemical indexes from 2019-nCoV infected patients linked to viral loads and lung injury. Sci China Life Sci 2020:63:364-74.

41. Marshall RP, Webb S, Bellingan GJ, Montgomery HE, Chaudhari B, McAnulty RJ, et al. Angiotensin converting enzyme insertion/deletion polymorphism is associated with susceptibility and outcome in acute respiratory distress syndrome. Am J Respir Crit Care Med 2002;166:64650.

42. Ling TY, Kuo MD, Li CL, Yu AL, Huang YH, Wu TJ, et al. Identification of pulmonary Oct-4+ stem/progenitor cells and demonstration of their susceptibility to SARS coronavirus (SARS-CoV) infection in vitro. Proc Natl Acad Sci USA 2006;103:9530-35.

43. Oudit GY, Penninger JM. Recombinant human angiotensin-converting enzyme 2 as a new renin-angiotensin system peptidase for heart failure therapy. Curr Heart Fail Rep 2011;8:176-83.

44. Gwathmey TM, Alzayadneh EM, Pendergrass KD, Chappell MC. Novel roles of nuclear angiotensin receptors and signaling mechanisms. Am J Physiol Regul Integr Comp Physiol 2012;302:R518-30.

45. Zhong J, Basu R, Guo D, Chow FL, Byrns S, Schuster M, et al. Angiotensin-converting enzyme 2 suppresses pathological hypertrophy, myocardial fibrosis, and cardiac dysfunction. Circulation 2010;122:717-28, 718 p following 728 .

46. Yamamoto K, Ohishi M, Katsuya T, Ito N, Ikushima M, Kaibe M, et al. Deletion of angiotensin-converting enzyme 2 accelerates pressure overload-induced cardiac dysfunction by increasing local angiotensin II. Hypertension 2006;47:718-26.

47. Nakamura K, Koibuchi N, Nishimatsu H, Higashikuni Y, Hirata Y, Kugiyama $\mathrm{K}$, et al. Candesartan ameliorates cardiac dysfunction observed in angiotensin-converting enzyme 2-deficient mice. Hypertens Res 2008;31:1953-61.

48. Kassiri Z, Zhong J, Guo D, Basu R, Wang X, Liu PP, et al. Loss of angiotensin-converting enzyme 2 accelerates maladaptive left ventricular remodeling in response to myocardial infarction. Circ Heart Fail 2009;2:446-55.
49. Oudit GY, Kassiri Z, Jiang C, Liu PP, Poutanen SM, Penninger JM, et al. SARS-coronavirus modulation of myocardial ACE2 expression and inflammation in patients with SARS. Eur J Clin Invest 2009;39:618-25.

50. Ye M, Wysocki J, William J, Soler MJ, Cokic I, Batlle D. Glomerular localization and expression of Angiotensin-converting enzyme 2 and Angiotensin-converting enzyme: implications for albuminuria in diabetes. J Am Soc Nephrol 2006;17:3067-75.

51. Soler MJ, Wysocki J, Batlle D. ACE2 alterations in kidney disease. Nephrol Dial Transplant 2013;28:2687-97.

52. Santos RA, Haibara AS, Campagnole-Santos MJ, Simoes e Silva AC, Paula $\mathrm{RD}$, Pinheiro SV, et al. Characterization of a new selective antagonist for angiotensin-(1-7), D-pro7-angiotensin-(1-7). Hypertension 2003;41:73743.

53. Prieto MC, Gonzalez-Villalobos RA, Botros FT, Martin VL, Pagan J, Satou $\mathrm{R}$, et al. Reciprocal changes in renal ACE/ANG II and ACE2/ANG 1-7 are associated with enhanced collecting duct renin in Goldblatt hypertensive rats. Am J Physiol Renal Physiol 2011;300:F749-55.

54. Wakahara S, Konoshita T, Mizuno S, Motomura M, Aoyama C, Makino $\mathrm{Y}$, et al. Synergistic expression of angiotensin-converting enzyme (ACE) and ACE2 in human renal tissue and confounding effects of hypertension on the ACE to ACE2 ratio. Endocrinology 2007;148:2453-7.

55. Wang G, Lai FM, Kwan BC, Lai KB, Chow KM, Li PK, et al. Expression of ACE and ACE2 in patients with hypertensive nephrosclerosis. Kidney Blood Press Res. 2011;34:141-9.

56. Park SE, Kim WJ, Park SW, Park JW, Lee N, Park CY, et al. High urinary ACE2 concentrations are associated with severity of glucose intolerance and microalbuminuria. Eur J Endocrinol 2013;168:203-10.

57. Chodavarapu H, Grobe N, Somineni HK, Salem ES, Madhu M, Elased $\mathrm{KM}$. Rosiglitazone treatment of type 2 diabetic $\mathrm{db} / \mathrm{db}$ mice attenuates urinary albumin and angiotensin converting enzyme 2 excretion. PLoS One 2013;8:e62833.

58. Xiao F, Zimpelmann J, Agaybi S, Gurley SB, Puente L, Burns KD. Characterization of angiotensin-converting enzyme 2 ectodomain shedding from mouse proximal tubular cells. PLoS One 2014;9:e85958.

59. Soler MJ, Wysocki J, Ye M, Lloveras J, Kanwar Y, Batlle D. ACE2 inhibition worsens glomerular injury in association with increased ACE expression in streptozotocin-induced diabetic mice. Kidney Int 2007;72:614-23.

60. Wysocki J, Ortiz-Melo DI, Mattocks NK, Xu K, Prescott J, Evora K, et al. ACE2 deficiency increases NADPH-mediated oxidative stress in the kidney. Physiol Rep 2014;2:e00264.

61. Zumla A, Hui DS, Azhar EI, Memish ZA, Maeurer M. Reducing mortality from 2019-nCoV: host-directed therapies should be an option. Lancet 2020;395:e35-6.

62. Du L, He Y, Zhou Y, Liu S, Zheng BJ, Jiang S. The spike protein of SARS-CoV--a target for vaccine and therapeutic development. Nat Rev Microbiol 2009;7:226-36.

63. Yang ZY, Kong WP, Huang Y, Roberts A, Murphy BR, Subbarao K, et al. A DNA vaccine induces SARS coronavirus neutralization and protective immunity in mice. Nature 2004;428:561-4.

64. Bisht H, Roberts A, Vogel L, Bukreyev A, Collins PL, Murphy BR, et al. Severe acute respiratory syndrome coronavirus spike protein expressed by attenuated vaccinia virus protectively immunizes mice. Proc Natl Acad Sci USA 2004;101:6641-6.

65. Chen Z, Zhang L, Qin C, Ba L, Yi CE, Zhang F, et al. Recombinant modified vaccinia virus Ankara expressing the spike glycoprotein of severe acute respiratory syndrome coronavirus induces protective neutralizing antibodies primarily targeting the receptor binding region. J Virol 2005;79:2678-88.

66. Kam YW, Kien F, Roberts A, Cheung YC, Lamirande EW, Vogel L, et al. Antibodies against trimeric $\mathrm{S}$ glycoprotein protect hamsters against SARS-CoV challenge despite their capacity to mediate FcgammaRIIdependent entry into B cells in vitro. Vaccine 2007;25:729-40.

67. He Y, Li J, Heck S, Lustigman S, Jiang S. Antigenic and immunogenic characterization of recombinant baculovirus-expressed severe acute 
respiratory syndrome coronavirus spike protein: implication for vaccine design. J Virol 2006;80:5757-67.

68. He Y, Zhou Y, Siddiqui P, Jiang S. Inactivated SARS-CoV vaccine elicits high titers of spike protein-specific antibodies that block receptor binding and virus entry. Biochem Biophys Res Commun 2004;325:445-52.

69. He Y, Zhu Q, Liu S, Zhou Y, Yang B, Li J, et al. Identification of a critical neutralization determinant of severe acute respiratory syndrome (SARS)associated coronavirus: importance for designing SARS vaccines. Virology 2005;334:74-82.

70. He Y, Zhou Y, Liu S, Kou Z, Li W, Farzan M, et al. Receptor-binding domain of SARS-CoV spike protein induces highly potent neutralizing antibodies: implication for developing subunit vaccine. Biochem Biophys Res Commun 2004;324:773-81.

71. Du L, Zhao G, He Y, Guo Y, Zheng BJ, Jiang S, et al. Receptor-binding domain of SARS-CoV spike protein induces long-term protective immunity in an animal model. Vaccine 2007;25:2832-8.

72. Du L, Zhao G, Lin Y, Chan C, He Y, Jiang S, et al. Priming with rAAV encoding RBD of SARS-CoV S protein and boosting with RBD-specific peptides for $\mathrm{T}$ cell epitopes elevated humoral and cellular immune responses against SARS-CoV infection. Vaccine 2008;26:1644-51.

73. Du L, He Y, Wang Y, Zhang H, Ma S, Wong CK, et al. Recombinant adenoassociated virus expressing the receptor-binding domain of severe acute respiratory syndrome coronavirus $S$ protein elicits neutralizing antibodies: Implication for developing SARS vaccines. Virology 2006;353:6-16.

74. Du L, Zhao G, Lin Y, Sui H, Chan C, Ma S, et al. Intranasal vaccination of recombinant adeno-associated virus encoding receptor-binding domain of severe acute respiratory syndrome coronavirus (SARS-CoV) spike protein induces strong mucosal immune responses and provides long-term protection against SARS-CoV infection. J Immunol 2008;180:948-56.

75. Qin C, Wang J, Wei Q, She M, Marasco WA, Jiang H, et al. An animal model of SARS produced by infection of Macaca mulatta with SARS coronavirus. J Pathol 2005;206:251-9.

76. He Y, Li J, Du L, Yan X, Hu G, Zhou Y, et al. Identification and characterization of novel neutralizing epitopes in the receptor-binding domain of SARS-CoV spike protein: revealing the critical antigenic determinants in inactivated SARS-CoV vaccine. Vaccine 2006;24:5498-508.

77. Walls AC, Tortorici MA, Snijder J, Xiong X, Bosch BJ, Rey FA, et al. Tectonic conformational changes of a coronavirus spike glycoprotein promote membrane fusion. Proc Natl Acad Sci USA 2017;114:11157-62.

78. Bosch BJ, van der Zee R, de Haan CA, Rottier PJ. The coronavirus spike protein is a class I virus fusion protein: structural and functional characterization of the fusion core complex. J Virol 2003;77:8801-11.

79. Li F. Structure, Function, and Evolution of Coronavirus Spike Proteins. Annu Rev Virol 2016;3:237-61.

80. Hu H, Li L, Kao RY, Kou B, Wang Z, Zhang L, et al. Screening and identification of linear B-cell epitopes and entry-blocking peptide of severe acute respiratory syndrome (SARS)-associated coronavirus using synthetic overlapping peptide library. J Comb Chem 2005;7:648-56.

81. Kruse RL. Therapeutic strategies in an outbreak scenario to treat the novel coronavirus originating in Wuhan, China. F1000Res 2020;9:72.

82. Han DP, Penn-Nicholson A, Cho MW. Identification of critical determinants on ACE2 for SARS-CoV entry and development of a potent entry inhibitor. Virology 2006;350:15-25.

83. Hofmann H, Geier M, Marzi A, Krumbiegel M, Peipp M, Fey GH, et al. Susceptibility to SARS coronavirus $\mathrm{S}$ protein-driven infection correlates with expression of angiotensin converting enzyme 2 and infection can be blocked by soluble receptor. Biochem Biophys Res Commun 2004;319:1216-21.
84. Adedeji AO, Severson W, Jonsson C, Singh K, Weiss SR, Sarafianos SG. Novel inhibitors of severe acute respiratory syndrome coronavirus entry that act by three distinct mechanisms. J Virol 2013;87:8017-28.

85. Huentelman MJ, Zubcevic J, Hernandez Prada JA, Xiao X, Dimitrov DS, Raizada MK, et al. Structure-based discovery of a novel angiotensinconverting enzyme 2 inhibitor. Hypertension 2004;44:903-6.

86. Vincent MJ, Bergeron E, Benjannet S, Erickson BR, Rollin PE, Ksiazek $\mathrm{TG}$, et al. Chloroquine is a potent inhibitor of SARS coronavirus infection and spread. Virol J 2005;2:69.

87. Wang M, Cao R, Zhang L, Yang X, Liu J, Xu M, et al. Remdesivir and chloroquine effectively inhibit the recently emerged novel coronavirus (2019-nCoV) in vitro. Cell Res 2020;30:269-71.

88. Gao J, Tian Z, Yang X. Breakthrough: Chloroquine phosphate has shown apparent efficacy in treatment of COVID-19 associated pneumonia in clinical studies. Biosci Trends 2020;14:72-3.

89. Ho TY, Wu SL, Chen JC, Li CC, Hsiang CY. Emodin blocks the SARS coronavirus spike protein and angiotensin-converting enzyme 2 interaction. Antiviral Res 2007;74:92-101.

90. Zhang XW, Yap YL. Old drugs as lead compounds for a new disease? Binding analysis of SARS coronavirus main proteinase with HIV, psychotic and parasite drugs. Bioorg Med Chem 2004;12:2517-21.

91. Tian X, Li C, Huang A, Xia S, Lu S, Shi Z, et al. Potent binding of 2019 novel coronavirus spike protein by a SARS coronavirus-specific human monoclonal antibody. Emerg Microbes Infect 2020;9:382-5.

92. Treml B, Neu N, Kleinsasser A, Gritsch C, Finsterwalder T, Geiger R, et al. Recombinant angiotensin-converting enzyme 2 improves pulmonary blood flow and oxygenation in lipopolysaccharide-induced lung injury in piglets. Crit Care Med 2010;38:596-601.

93. Haschke M, Schuster M, Poglitsch M, Loibner H, Salzberg M, Bruggisser $\mathrm{M}$, et al. Pharmacokinetics and pharmacodynamics of recombinant human angiotensin-converting enzyme 2 in healthy human subjects. Clin Pharmacokinet 2013;52:783-92.

94. Khan A, Benthin C, Zeno B, Albertson TE, Boyd J, Christie JD, et al. A pilot clinical trial of recombinant human angiotensin-converting enzyme 2 in acute respiratory distress syndrome. Crit Care 2017; 21: 234.

95. Deng W, Deng Y, Deng J, Wang DX, Zhang T. Losartan attenuated lipopolysaccharide-induced lung injury by suppression of lectin-like oxidized low-density lipoprotein receptor-1. Int J Clin Exp Pathol 2015;8:15670-6.

96. Zambelli V, Bellani G, Borsa R, Pozzi F, Grassi A, Scanziani M, et al. Angiotensin-(1-7) improves oxygenation, while reducing cellular infiltrate and fibrosis in experimental Acute Respiratory Distress Syndrome. Intensive Care Med Exp 2015;3:44.

97. Klein N, Gembardt F, Supe S, Kaestle SM, Nickles H, Erfinanda L, et al. Angiotensin-(1-7) protects from experimental acute lung injury. Crit Care Med 2013;41:e334-43.

98. Kulemina LV, Ostrov DA. Prediction of off-target effects on angiotensinconverting enzyme 2. J Biomol Screen 2011;16:878-85.

99. Shenoy V, Gjymishka A, Jarajapu YP, Qi Y, Afzal A, Rigatto K, et al. Diminazene attenuates pulmonary hypertension and improves angiogenic progenitor cell functions in experimental models. Am J Respir Crit Care Med 2013;187:648-57.

How to cite this article: Liu M, Wang T, Zhou Y, Zhao Y, Zhang Y, Li J. Potential role of ACE2 in coronavirus disease 2019 (COVID-19) prevention and management. J Transl Int Med 2020; 8: 9-19. 